\title{
The Seroprevalence of Anti-Hepatitis E IgG Antibodies in Blood Donors in The Greater Romagna Area, Italy
}

\author{
Anna Pierro ${ }^{1}$, Simona Semprini ${ }^{1}$, Michela Fantini ${ }^{1}$, Patrizia Billi ${ }^{1}$, Rino Biguzzi ${ }^{2}$, Vanessa Agostini², Vittorio Sambri1,3 \\ 1 Unit of Microbiology, The Greater Romagna Hub Laboratory, Pievesestina, Italy \\ 2 Unit of Immunohematology and Transfusion Medicine, The Greater Romagna Hub Laboratory, Pievesestina, Italy \\ 3 Department of Experimental, Diagnostic and Specialty Medicine, University of Bologna, Bologna, Italy
}

\begin{abstract}
Objective: Data about Hepatitis Evirus (HEV) prevalence arevariablein Europeand alsolimited in Italy. We aimed to describe the seroprevalence of immunoglobulin G (IgG) antibodies against HEV infection among blood donors (BDs) in the Greater Romagna area, North-Eastern Italy. Materials and Methods: All serum samples were at first screened for anti-HEV IgG antibodies by a commercial enzyme-linked immunosorbent assay (ELISA) (DIA.PRO Diagnostic Bioprobes, Sesto San Giovanni (MI), Italy), used in our diagnostic routine and so considered as the reference test. Then, all serum samples were re-tested for anti-HEV IgG antibodies by the other two commercial ELISA (Wantai, Biologic Pharmacy Enterprise, Beijing, China; Euroimmun, Lübeck, Germany). The seroprevalence rate was calculated based on the immunoblotting (IB) assay confirmation.

Results: A total of 500 BDs samples were tested by three commercial ELISA tests; positive and borderline results were confirmed by an IB assay. The overall anti-HEV IgG prevalence was 3.8\% (CI: $2.303-5.871$ ), 1.6\% (CI: 0.693 - 3.128) and 2.8\% (CI: 1.539 - 4.653) when samples were tested with DIA.PRO, Euroimmun and Wantai methods, respectively. The seroprevalence of IgG antibodies against HEV infection was found to be similar or lower than the previous reports in Italy. The results obtained by the different ELISA tests were similar.

Conclusion: The data obtained in this study underline the need for achieving a harmonised testing algorithm that is necessary in order to be able to compare data from different studies. We suggest that the identification of potential HEV human infection should be added to the standard laboratory work-up for viral hepatitis.
\end{abstract}

\section{INTRODUCTION}

H epatitis E virus (HEV) represents one of the major cause of acute viral hepatitis worldwide (1), according to the WHO, one-third of the world population has been exposed to HEV (2). In Europe, there is extensive evidence indicating that HEV is prevalently transmitted by the ingestion of pork and wild boar raw meat (3), however, other sources of infection such as blood transfusion and organ transplantations have also been reported $(4,5)$.
Corresponding Author: Anna Pierro

E-mail: anna.pierro@auslromagna.it

Received: August 5, 2019 Accepted: October 23, 2019 Published: November 26, 2019

\section{Suggested citation:}

Pierro A, Semprini S, Fantini M, Bill P, Biguzzi R, Agostini $V$ et al. The seroprevalence of anti-hepatitis $\mathrm{E}$ IgG antibodies in blood donors in the Greater Romagna area, Italy. Infect Dis Clin Microbiol 2019; 3: 123-127.

DOI: $10.36519 / \mathrm{idcm} .2019 .19017$ 
Sporadic and autochthonous cases of HEV infection in developed countries, including Europe, were reported among the patients who had no history of travelling to hepatitis E endemic countries, therefore HEV is currently considered as an emerging pathogen in these developed areas (6). HEV seroprevalence shows significant variability in different European regions, ranging from 0,03\% to 52\% among the general and blood donors (BDs) populations $(7,8)$. In Italy, data about HEV prevalence in general population and BDs are scattered and variably available (9-14). A study published in 2016 reported a seroprevalence of $49 \%$ among BDs in an area close to the one included in this study (8). The anti HEV antibody seroprevalence varies greatly depending on the area studied and the tests used. This study aimed to assess the HEV infection prevalence among BDs living in the Grater Romagna area, in North-Eastern Italy. The Greater Romagna area includes the provinces of Forli-Cesena, Ravenna and Rimini with a total of 32,000 BDs. Moreover, using different serological test systems significantly influences the estimates of seroprevalence (15). A secondary goal of this study was to evaluate the variability of seroprevalence results obtained on the same group of patients when different serological methods are used.

\section{MATERIALS AND METHODS}

All serum samples were at first screened for anti-HEV immunoglobulin G (IgG) antibodies by a commercial enzyme-linked immunosorbent assay (ELISA) (DIA.PRO Diagnostic Bioprobes, Sesto San Giovanni (MI), Italy), used in our diagnostic routine and so considered as the reference test. Then, all serum samples were re-tested for anti-HEV IgG antibodies by the other two commercial ELISA (Wantai, Biologic Pharmacy Enterprise, Beijing, China; Euroimmun, Lübeck, Germany). The obtained results were used to calculate the agreement between the different tests. All the samples that resulted positive and borderline by anyone of the three ELISA methods used were confirmed by an IgG immunoblotting (IB) assay (recomLine HEV IgG/IgM Mikrogen Diagnostik, Germany), which is currently used as a confirmatory test in our diagnostic routine. The seroprevalence rate was calculated based on the IB confirmed test results.
The sample size is representative of the BDs population in this area, and the sample sizes were calculated considering the expected prevalence of HEV infection. Samples were randomly daily collected among BDs screened by the Blood Transfusion Unit and tested for the aim of this study by the Unit of Microbiology of the Greater Romagna Hub Laboratory, Pievesestina, Italy. The prevalence of anti-HEVIgG antibodies was calculated for each one of the screening tests used, as well as the percentages of sensitivity and specificity were calculated (Table 1). The proportion of agreement between the different ELISA tests was detected by using Cohen's Kappa coefficient that assumes values between -1 (no agreement) and 1 (complete agreement). Statistical analysis was performed using Stata software version 14.2 (Stata Corp., College Station, TX, USA). Statistical significance was set as $\mathrm{p}<0.05$.

\section{RESULTS}

From May 1st to July 31st 2016, 90 serum samples were collected from Forlì-Cesena BDs, 300 from Ravenna BDs and 110 from Rimini BDs, for a total of 500 BDs (78\% male, age: $18-82$ years, median 43 years). In 22 out of 500 (4\%) serum samples tested, anti-HEV IgG was found to be positive by DIA.PRO, and 19 of these 22 positive samples were further confirmed by IB test. When the 500 serum samples were tested by the Euroimmun assay, in 11 Anti-HEV

\section{HIGHLIGHTS}

- The overall anti-HEV IgG prevalence was $3.8 \%$ in North-Eastern Italy.

- The seroprevalence of IgG antibodies against HEV infection was found to be similar or lower than the previous reports in Italy.

- The results obtained by the different ELISA tests were similar.

- The data obtained in this study underline the need for achieving a harmonised testing algorithm that is necessary in order to be able to compare data from different studies.

- We suggest that the identification of potential HEV human infection should be added to the standard laboratory work-up for viral hepatitis. 
Table 1. Seroprevalence results: The Table summarizes the different percentage of seroprevalence calculated on EIA positive and on IB confirmed samples for each one of the ELISA method used. Specificity and sensitivity values were calculated using the DIA.PRO ELISA arbitrarily as the reference test.

\begin{tabular}{|l|l|l|l|l|}
\hline & $\begin{array}{l}\text { The seroprevalence of anti-HEV } \\
\text { IgG before confirmatory testing } \\
\mathbf{n}=500\end{array}$ & $\begin{array}{l}\text { The seroprevalence of anti-HEV } \\
\text { IgG after confirmatory IB testing } \\
\mathrm{n}=500\end{array}$ & Sensitivity & Specificity \\
\hline DIA.PRO & $4.2 \%$ & $3.8 \%$ & $86.4 \%$ & $100 \%$ \\
\hline EUROIMMUN & $2.2 \%$ & $1,6 \%$ & $72.7 \%$ & $100 \%$ \\
\hline WANTAI & $3,4 \%$ & $2,8 \%$ & $82.3 \%$ & $100 \%$ \\
\hline
\end{tabular}

(a)

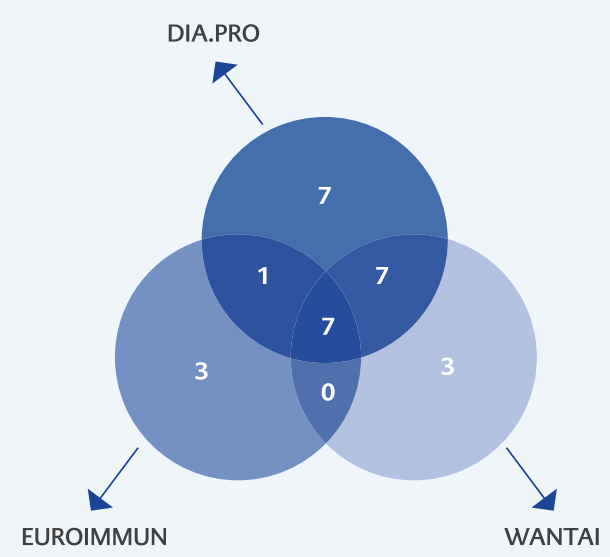

(b)

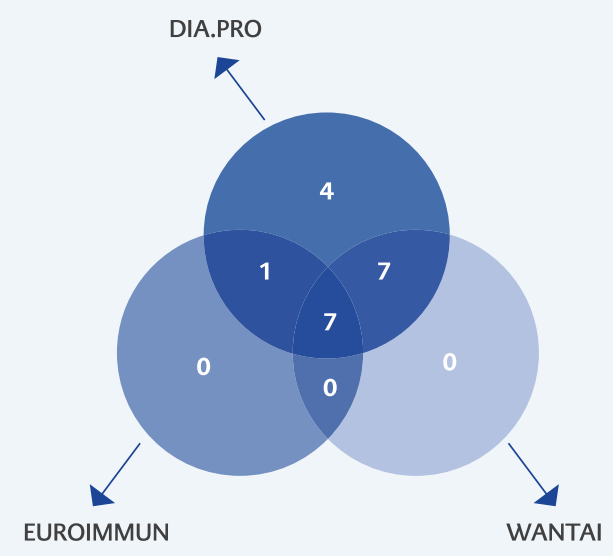

Figure 1. Venn diagram: a) distribution of IgG-HEV positive serum sample according to the three ELISA (DIA.PRO, EUROIMMUN, WANTAI) methods, and b) after confirmation by IB. The number in selected intersected area of individual diagrams indicate the specimens identified as positive by each assay.

IgG was found to be positive (2\%), and Eight out of these 11 IgG positive specimens were confirmed by IB. By using Wantai method, in 17 samples out of 500 (3.4\%), Anti-HEV IgG was found to be positive, and 14 IgG positive samples were confirmed by IB.

The eight positive samples obtained by the Euroimmune method and confirmed with IB, also the 14 samples obtained with Wantai and confirmed with IB were all included in the 19 specimens initially selected by DIA PRO (with IB confirmation) (Figure 1). The overall anti-HEV IgG prevalence was 3.8\% (CI: $2.303-5.871$ ), $1.6 \%$ (CI: $0.693-3.128$ ) and $2.8 \%$ (CI: 1.539 - 4.653) when samples were tested with DIA. PRO, Euroimmun and Wantai methods, respectively. Table 1 summarizes the different values of seroprevalence calculated for individual serologic tests before and after the IB confirmatory testing.

Furthermore, the comparison between the DIA.PRO method (arbitrarily selected as the reference being the one in routine use in our laboratory) versus the Euroimmun, and the DIA.PRO versus the Wantai assays showed a concordance of $98 \%$ and $99 \%$, respectively.

\section{DISCUSSION}

This study aimed to describe the IgG seroprevalence against HEV infection in a BDs population living in the Greater Romagna area: the overall percentage of seroprevalence was $3.8 \%$. This value has been obtained with the diagnostic algorithm routinely used in our laboratory (based on DIA.PRO EIA test followed by IB confirmation). However, the rates of seroprevalence in this BDs population calculated 
as above reported for each one of the methods used in this study are quite close, ranging from $3.8 \%$ and $1.6 \%$ (Table 1).

The seroprevalence values found in this study were similar or even lower than those already published among other different Italian BDs population $[9,11,13]$. A recent study performed among Italian BDs reported an overall IgG-HEV prevalence of $8.7 \%$ showing this as the lowest seroprevalence rate reported among BDs population in Europe. In particular, in the Emilia Romagna region, it has been reported an overall IgG seroprevalence of $3.6 \%$, similarly to that values found in our study (that included only the Romagna area of the Emilia-Romagna region) by using the Wantai method [14]. Nevertheless, a work published in 2016 describing the HEV seroprevalence in a quite geographically close area to the one included in this report (the Abruzzo region, some $250 \mathrm{~km}$ South of the studied zone) showed anti-HEV antibodies' overall prevalence of $49 \%$ among an unselected population of BDs [8]. As far as the detection of anti-HEV IgG is concerned, the results obtained in this study by the different commercially available ELISA tests are similar. As previously reported, the use of a confirmatory test based on the IB method does not substantially modify the seroprevalence values [15]. The results obtained are in strong disagreement with those reported in other studies that considered Wantai as- say as the reference method for the investigation of the HEV human seroprevalence. Most of the studies using the Wantai test are compared and analysed in the publication from Hartl and co-workers: this work showed higher seroprevalence values obtained by using this Chinese test when compared to other serological methods [16].

Epidemiological studies in the last few years have demonstrated that HEV infection should be considered as an emerging zoonosis. However, data about the human seroprevalence are extremely variable and largely depending on many issues, including the geographical area, population studied, and, in particular, on the serological assay used (17). Moreover, the public health implications of the HEV infection in Europe have changed because of the increasing numbers of cases and the recent report of chronic, persistent HEV infection associated with progression to cirrhosis in immune-suppressed patients (18).

In conclusion, the data obtained in this study and the overall trend of HEV infection in Europe and Italy underline the need for achieving a harmonised testing algorithm that is necessary in order to be able to compare data from different studies. We suggest that the identification of potential HEV human infection should be added to the standard laboratory work-up for viral hepatitis.
Peer-review: Externally peer-reviewed

Author Contributions: Concept - A.P., V.S.; Design - A.P.; Supervision - V.S.; Materials - R.B., V.A.; Data Collection and/ or Processing - P.B., S.S; Analysis and/or Interpretation - M.F.; Literature Review - A.P.; Writer - A.P.; Critical Reviews - V.S;
Conflict of Interest: The authors have no conflict of interest to declare.

Financial Disclosure: The authors declared that this study has received no financial support.

\section{REFERENCES}

1 Capai L, Charrel R, Falchi A. Hepatitis E in high-income countries: What do we know? And what are the knowledge gaps? Viruses 2018; 10.

2 Khuroo MS, Khuroo MS. Hepatitis E: an emerging global disease-from discovery towards control and cure. J Viral Hepat 2016; 23: 68-79.
3 Domanović D, Tedder R, Blümel J, Zaaijer H, Gallian P, Niederhauser C, et al. Hepatitis E and blood donation safety in selected European countries: a shift to screening? Euro Surveill 2017; 22.

4 Pérez-Gracia MT, García M, Suay B, Mateos-Lindemann ML. Current Knowledge on Hepatitis E. J Clin Transl Hepatol 2015; 3: 117-26. 
5 Schlosser B, Stein A, Neuhaus R, Pahl S, Ramez B, Krüger DH, et al. Liver transplant from a donor with occult HEV infection induced chronic hepatitis and cirrhosis in the recipient. J Hepatol 2012; 56: 500-2.

6 Sayed IM, Vercouter AS, Abdelwahab SF, Vercauteren K, Meuleman P. Is hepatitis E virus an emerging problem in in dustrialized countries? Hepatology 2015; 62: 1883-92.

7 Clemente-Casares P, Ramos-Romero C, Ramirez-Gonzalez E, Mas A. Hepatitis E virus in industrialized countries: the silent threat. Biomed Res Int 2016; 2016: 9838041.

8 Lucarelli C, Spada E, Taliani G, Chionne P, Madonna E, Marcantonio $\mathrm{C}$, et al. High prevalence of anti-hepatitis $\mathrm{E}$ virus antobodies among blood donors in central Italy, February to March 2014. Euro Surveill 2016; 21.

9 Zanetti AR, Dawson GJ. Hepatitis type E in Italy: a seroepidemiological survey. J Med Virol 1994, 42: 318-20.

10 Vulcano A, Angelucci M, Candelori E, Martini V, Patti AM, Mancini C, et al. HEV prevalence in the general population and among workers at zoonotic risk in Latium Region. Ann Ig 2007; 19: 181-6.

11 Masia G, Orrù G, Liciardi M, Desogus G, Coppola RC, Murru V, et al. Evidence of hepatitis E virus (HEV) infection in human and pigs in Sardinia, Italy. J Prev Med Hyg 2009, 50: 227-31.

12 Scotto G, Martinelli D, Centra M, Querques M, Vittorio F, Delli Carri P, et al. Epidemiological and clinical features of HEV infection: a survey in the district of Foggia (Apulia, Southern Italy). Epidemiol Infect 2014; 142: 287-94.

13 Puttini C, Riccio ML, Redi D, Tordini G, Cenerini M, Romanello F, et al. Seroprevalence of hepatitis E virus (HEV) infection in blood donors and renal transplant recipients: a retrospective study from central Italy. Infez Med 2015, 23: 253-6.

14 Spada E, Pupella S, Pisani G, Bruni R, Chionne P, Madonna E, et al. A nationwide retrospective study on prevalence of hepatitis E virus infection in Italian blood donors. Blood Transfus 2018, 16: 413-21.

15 Avellon A, Morago L, Garcia-Galera del Carmen M, Munoz M, Echevarría JM. Comparative sensitivity of commercial tests for hepatitis E genotype 3 virus antibody detection. J Med Virol 2015, 87: 1934-9.

16 Hartl J, Otto B, Madden RG, Webb G, Woolson KL, Kriston L, et al. Hepatitis E seroprevalence in Europe: a meta-analysis. Viruses 2016; 8: 211.

17 Lapa D, Capobianchi MR, Garbuglia AR. Epidemiology of hepatitis e virus in European countries. Int J Mol Sci 2015, 16: 25711-43.

18 Mellgren A, Karlsson M, Karlsson M, Lagging M, Wejstål R, Norder H. High seroprevalence against hepatitis E virus in patients with chronic hepatitis C virus infection. J Clin Virol 2017, 88: $39-45$ 